\title{
Power Spectral Density Specification and Analysis of Large Optical Surfaces
}

\author{
Erkin Sidick \\ Jet Propulsion Laboratory, California Institute of Technology, 4800 Oak Grove Drive, Pasadena, \\ CA, USA 91109
}

\begin{abstract}
The 2-dimensional Power Spectral Density (PSD) can be used to characterize the mid- and the high-spatial frequency components of the surface height errors of an optical surface. We found it necessary to have a complete, easy-to-use approach for specifying and evaluating the PSD characteristics of large optical surfaces, an approach that allows one to specify the surface quality of a large optical surface based on simulated results using a PSD function and to evaluate the measured surface profile data of the same optic in comparison with those predicted by the simulations during the specification-derivation process. This paper provides a complete mathematical description of PSD error, and proposes a new approach in which a 2-dimensional (2D) PSD is converted into a 1-dimensional (1D) one by azimuthally averaging the 2D-PSD. The 1D-PSD calculated this way has the same unit and the same profile as the original PSD function, thus allows one to compare the two with each other directly.
\end{abstract}

Keywords: Power spectral density, wavefront, optical surface specification, large optical surfaces

\section{INTRODUCTION}

Some space optical systems such as Terrestrial Planet Finder Coronagraph (TPF-C) require large optics with very high surface quality ${ }^{1-2}$. The developmental cycle of such an optical system includes, but is not limited to, the following two steps: (i) Deriving requirements (or specifications) on the surface quality of individual optical components before they are fabricated and (ii) evaluating the quality of those components against the pre-determined specifications after they are fabricated. One of the preferred metrics used for specifying surface roughness is the power spectral density (PSD). It is computed from the amplitude of spatial frequency components present in the Fourier spectrum of the surface height of an optical component, and has been used for some time in optics mainly for specifying high spatial frequency characteristics of optical surfaces to quantify their scattering properties ${ }^{3-5}$. More recently, an optical metric referred to as "PSD error" has started to be used by optical engineers to describe the quality of optical surfaces and wavefront in optical systems. The surface height or surface roughness data can be obtained from measurements of the bidirectional reflectance distribution function, from surface profiles measured using an optical or mechanical profiler, or from surface height maps measured using an interferometer such as Zygo-interferometer. This paper is about the processing of 2dimensional surface height data measured interferometrically. When an optic has a circular aperture, the PSD can be used to characterize the mid- and the high-spatial frequency components of its surface height. In such a case, the low frequency surface error (or figure error) of the optic can be analyzed with Zernike polynomials. If an optic has an irregularly shaped aperture, one must rely on the PSD analysis to characterize its surface quality since Zernike polynomials are an orthogonal set for circular apertures only.

Several different approaches have been reported on how to calculate PSD from surface height measurements, ${ }^{1,5-7}$. However, when trying to derive a PSD specification on a large space optic, we failed to find a reference that addresses the following two issues simultaneously: Deriving PSD requirements on an optic based on a PSD function and characterizing the same optic after it was made in terms of those pre-determined PSD specifications. This paper is intended to fill such a gap. Specifically, we will provide a complete approach for specifying and evaluating the PSD characteristics of large optical surfaces. We will propose a new method for converting a 2D-PSD into a 1D-PSD by azimuthally averaging the former. We will show that the 1D-PSD calculated this way can be directly compared with the PSD function used to derive the original PSD specifications, and eliminates the need for variance-reducing procedures such as using windowing filters (such as Hann or Welch windows), zero-padding and averaging multiple subaperture

Modeling Aspects in Optical Metrology II, edited by Harald Bosse, Bernd Bodermann, Richard M. Silver, Proc. of SPIE Vol. 7390, 73900L · C 2009 SPIE · CCC code: 0277-786X/09/\$18 · doi: 10.1117/12.823844 
PSDs. The key surface error metrics we chose to use in our specifications are low-order Zernikes, the band-limited RMS error of mid- and high-spatial frequency components and the exponent of an inverse power law of a PSD function.

\section{MATHEMATICAL DESCRIPTION}

\subsection{Calculation of PSD from Surface Height Data}

In this subsection, we describe the mathematical model we have used for obtaining a 2D- PSD from surface height data. We will describe only the essential part of the basic PSD theory, and more detailed description can be found in other literature, see, for example, Ref. 7. We provide both the integral and the digitized forms of the formulation whenever we see appropriate. The fixed-length Fourier-transform of a surface height map $h(x, y)$ is defined as

$$
\begin{aligned}
& \widetilde{H}(u, v)=\int_{-b / 2}^{b / 2} \int_{-a / 2}^{a / 2} h(x, y) e^{-2 \pi j(u x+v y)} d x d y, \\
& \widetilde{H}\left(u_{m}, v_{n}\right)=\mathfrak{S}\left\{h\left(x_{m}, y_{n}\right)\right\} \Delta x \Delta y,
\end{aligned}
$$

where $x$ and $y$ are the surface position variables in the horizontal and the vertical directions, and $u$ and $v$ are the spatial frequency variables in the horizontal and the vertical directions, respectively. In the following, we will also use a radial frequency variable $\rho$, which is defined as $\rho=\sqrt{u^{2}+v^{2}}$. The $\mathfrak{I}$ symbol in Eq. (2) represents a 2-Dimensional Fast Fourier-Transform (FFT) routine. We chose not to give the FFT expression explicitly here because nowadays everyone uses the built-in FFT routine included in various types of commercial software, such as the fft2.m function in MATLAB, to perform an FFT operation on a matrix. The parameters $a$ and $b$ are the total width and the total height of the surface height map. The 2D-PSD is defined as the squared amplitude per unit area of the spectrum of a surface height map. Thus it can be expressed as

$$
\begin{gathered}
\operatorname{PSD}(u, v)=\frac{1}{A}|\widetilde{H}(u, v)|^{2}=\frac{1}{a b}\left|\int_{-b / 2}^{b / 2} \int_{-a / 2}^{a / 2} h(x, y) d x d y\right|^{2}, \\
P S D\left(u_{m}, v_{n}\right)=\frac{1}{A}\left|\widetilde{H}\left(u_{m}, v_{n}\right)\right|^{2}=\frac{(\Delta x \Delta y)^{2}}{A}\left|\Im\left\{h\left(x_{m}, y_{n}\right)\right\}\right|^{2}=\frac{A}{(M N)^{2}}\left|\Im\left\{h\left(x_{m}, y_{n}\right)\right\}\right|^{2} .
\end{gathered}
$$

Indices $m$ and $n$ take on values from 1 to $M$ and 1 to $N$, where $M$ and $N$ are the numbers of sample points in $x$ and $y$, respectively. We have utilized the following relation in arriving at the last expression in Eq. (4):

$$
A=a b=(M \Delta x)(N \Delta y) .
$$

As we can see from Eq. (4), the $\operatorname{PSD}\left(u_{m}, v_{n}\right)$ has a unit of $(\text { length })^{2}(\text { length })^{2}$. For large optics as in our case, one can choose $(n m)^{2}(m)^{2}$. Other relationships among the various variables in Eq. (4) are

$$
\begin{aligned}
& \Delta x=a / M \\
& \Delta y=b / N \\
& x_{m}=[m-(M+1) / 2] \Delta x, \quad m=1,2, \ldots, M \\
& y_{n}=[n-(N+1) / 2] \Delta y, \quad n=1,2, \ldots, N \\
& u_{m}=[m-(M+1) / 2] /(M \Delta x), \quad m=1,2, \ldots, M \\
& v_{n}=[n-(N+1) / 2] /(N \Delta y), \quad n=1,2, \ldots, N
\end{aligned}
$$

One of the quantities widely used in PSD specification is the root-mean-square (RMS) error, $\sigma$. It can be calculated either from the surface height $h(x, y)$ or from the 2D-PSD as follows: 


$$
\begin{gathered}
\sigma^{2}=\frac{1}{A} \int_{-b / 2}^{b / 2} \int_{-a / 2}^{a / 2}[h(x, y)]^{2} d x d y=\int_{-1 / 2 \Delta y}^{1 / 2 \Delta y} \int_{-1 / 2 \Delta x}^{1 / 2 \Delta x} P S D(u, v) d u d v, \\
\sigma^{2}=\frac{\Delta x \Delta y}{A} \sum_{n=1}^{N} \sum_{m=1}^{M}\left[h\left(x_{m}, y_{n}\right)\right]^{2}=\frac{1}{A} \sum_{n=1}^{N} \sum_{m=1}^{M} P S D\left(u_{m}, v_{n}\right) .
\end{gathered}
$$

This is a bandwidth limited RMS value of the PSD error, as the magnitude of the highest surface spatial frequency resolved in the measurement process is the Nyquist frequency $u_{c}=1 / 2 \Delta x$ or $v_{c}=1 / 2 \Delta y$. If the mean of $h\left(x_{m}, y_{n}\right)$ is removed before calculating $\sigma^{2}$ using Eq. (8), then the resultant $\sigma^{2}$ value corresponds to the mean-square roughness of the surface ${ }^{5}$. Note that Eqs. (7) and (8) correspond to a general expression of Parseval's theorem and can be used as a check on the validity of the methods used to calculate the 2D-PSD. Note also that $1 / A$ in the last expression of Eq. (8) can be replaced with $(\Delta u)(\Delta v)$ based on the relationships given in Eq. (6), where $\Delta u=1 / M \Delta x$ and $\Delta v=1 / N \Delta y$ are the data point intervals (or pixel widths) in the spatial frequency domain.

In most cases, optical surfaces have circular clear apertures. In such a case, the RMS value of the surface height $h\left(x_{m}, y_{n}\right)$ needs to be calculated from only those data points inside its clear aperture. That is,

$$
\sigma_{\text {aper }}=\sqrt{\frac{1}{K} \sum_{k=1}^{K}\left[h\left(r_{m n}\right)\right]^{2}}, r_{m n}=\sqrt{x_{m}^{2}+y_{n}^{2}} \leq D,
$$

where $\mathrm{K}$ is the total number of data points that satisfy $r_{m n}=\sqrt{x_{m}{ }^{2}+y_{n}{ }^{2}} \leq D, D$ is the diameter of the clear aperture, and subscript "aper" means "aperture". For many applications, the location of the PSD error on the $u v$-plane is not important. Thus, a PSD specification can be written in one dimension. So far a 1D-PSD has been obtained either by slicing the 2D-PSD along a particular axis, or by integrating the 2D-PSD along $u$ - or $v$-axis or azimuthally ${ }^{1,7,8}$. Here we propose an alternative approach: We average $\operatorname{PSD}\left(u_{m}, v_{n}\right)$ azimuthally to obtain one PSD value from the data in every small annular region on the 2D-PSD map. We will explain the advantage of this approach in the following section. We do this averaging in the following way. (1) Express $\operatorname{PSD}\left(u_{m}, v_{n}\right)$ as a function of $\rho_{m n}=\sqrt{u_{m}{ }^{2}+v_{n}{ }^{2}}$ in the polar coordinates and obtain $P S D\left(\rho_{m n}\right)$. (2) Sort $\rho_{m n}$ from minimum to maximum to obtain $\rho_{l}$ and $P S D\left(\rho_{l}\right)$, where $l=1,2, \ldots, L=M+N$. (3) Divide $\operatorname{Max}\left(\rho_{l}\right)=\rho_{L}$ into $Q-1$ intervals in a log-space with end points $\rho_{q}^{\prime}, q=2,3, \ldots, Q$. (4) Calculate the radially- or azimuthally-averaged $Q-1$ pairs of $\bar{\rho}_{q}-\bar{P}_{q}$ data points, and set $\bar{\rho}_{1}-\bar{P}_{1}$ to their values at the center of $P S D\left(u_{m}, v_{n}\right)$ map. The $\bar{P}_{q}$ obtained this way is the 1D-PSD. For example, assume the $5^{\text {th }}$ interval satisfying $\rho_{5}^{\prime} \leq \rho_{l}<\rho_{6}^{\prime}$ has 100 pairs of $\rho_{l}-\operatorname{PSD}\left(\rho_{l}\right)$ data points from $l=401$ to $l=500$. Then the radially averaged $\bar{\rho}_{5}-\bar{P}_{5}$ pair is obtained as

$$
\begin{aligned}
& \bar{\rho}_{5}=\frac{1}{100} \sum_{l=401}^{l=500} \rho_{l} \\
& \bar{P}_{5}=1 \mathrm{D}-P S D=\frac{1}{100} \sum_{l=401}^{l=500} P S D\left(\rho_{l}\right)
\end{aligned}
$$

\subsection{Synthesizing Surface Height Maps from a PSD Function}

As mentioned in the introduction, one needs to rely exclusively on synthesized surface heights or simulated PSD errors during the specification-derivation phase of an optical project. We used the following Lorentzian equation as our PSD function $^{1,4,9}$ : 


$$
P S D^{\prime}\left(u_{m}, v_{n}\right)=\frac{\sigma_{0}^{2} A}{h_{0}} g\left(\rho_{m n}, p\right)=\frac{\sigma_{0}^{2} A}{h_{0}} \frac{1}{1+\left(\rho_{m n} / \rho_{H P}\right)^{p}},
$$

where

$$
h_{0}=\sum_{m=1}^{M} \sum_{n=1}^{N} \frac{1}{1+\left(\rho_{m n} / \rho_{H P}\right)^{p}}
$$

is a normalization factor, $\rho_{H P}$ is the half-power frequency, and $p$ is the exponent of the inverse power-law. We used a primed parameter name for PSD in Eq. (11) to distinguish it from that in Eq. (4). That is, the PSD function in Eq. (11) is used for surface height synthesis, whereas the one in Eq. (4) is used for surface height analysis, and they are usually not the same as will be explained below. The factor $\left(\sigma_{0}^{2} A / h_{0}\right)$ in Eq. (10) is chosen such that the resultant surface height, $h^{\prime}\left(x_{m}, y_{n}\right)$, always has a RMS value of $\sigma_{0}$ when (i) both $M$ and $N$ are even integers ( $M=N$ and $a=b$ in most cases) and (ii) $h^{\prime}\left(x_{m}, y_{n}\right)$ is not multiplied by a circular mask defining the clear aperture of the corresponding optical surface to obtain $h\left(x_{m}, y_{n}\right)$. That is, $h^{\prime}\left(x_{m}, y_{n}\right)$ and $h\left(x_{m}, y_{n}\right)$ are related by

$$
h\left(x_{n}, y_{m}\right)=h^{\prime}\left(x_{n}, y_{m}\right) \times \operatorname{mask}\left(x_{n}, y_{m}\right),
$$

where $\operatorname{mask}\left(x_{n}, y_{m}\right)=1$ inside the clear aperture of the optical surface and $\operatorname{mask}\left(x_{n}, y_{m}\right)=0$ otherwise.

It is now straightforward to calculate $h^{\prime}\left(x_{m}, y_{n}\right)$ from the $P S D^{\prime}\left(u_{m}, v_{n}\right)$ in Eq. (11). That is, based on what is described in Eq. (4), we have

$$
h^{\prime}\left(x_{n}, y_{m}\right)=\frac{1}{\Delta x \Delta y} \mathfrak{J}^{-1}\left\{\mathrm{e}^{i \phi\left(u_{n}, v_{m}\right)} \sqrt{A \times P S D^{\prime}\left(u_{n}, v_{m}\right)}\right\},
$$

where $\mathfrak{J}^{-1}$ represents an inverse-FFT algorithm, such as the ifft2.m function in MATLAB, and $\phi\left(u_{m}, v_{n}\right)$ is an antisymmetric, randomly-valued phase map satisfying $-\pi \leq \phi\left(u_{m}, v_{n}\right) \leq \pi$. Because the $h\left(x_{m}, y_{n}\right)$ in Eq. (4) is real, its Fourier-transform has the following symmetry property: $\widetilde{H}\left(u_{m}, v_{n}\right)=\widetilde{H}^{*}\left(-u_{m},-v_{n}\right)$, where “*” denotes complexconjugate. Thus the anti-symmetric condition required on $\phi\left(u_{m}, v_{n}\right)$ guarantees that the resulted surface height $h^{\prime}\left(x_{m}, y_{n}\right)$ is real. The realization of such a $\phi\left(u_{m}, v_{n}\right)$ matrix is also straightforward in MATLAB. For example, one can obtain such a phase map by calculating the phase of the spectrum of $r\left(u_{m}, v_{n}\right)$, an $M \times N$ random number matrix having either a uniform or a normal distribution. We found that, if $M=N=$ even integers, the center of $\phi\left(u_{m}, v_{n}\right)$, or $\phi(0,0)$, is located at $n=m=N / 2+1$ (after shifting the zero-frequency component to the center of spectrum by using, for example, fftshift.m algorithm in MATLAB). Also, $\phi(0,0)=0$ if $r\left(u_{m}, v_{n}\right)$ has a uniform distribution, and $\phi(0,0)=\pi$ if it has a normal distribution.

It should be noted that the PSD error obtained from Eq. (11) does not compute phase information, so it does not specify a unique surface height or wavefront map. Rather, it specifies a family of uncorrelated surface height maps. For that reason, when determining the controllability of the PSD errors in an optical system using surface-deforming actuators ${ }^{\mathbf{1 0}}$, we carry out wavefront control simulations on at least 50 realizations of PSD errors for each $\sigma$-value category to capture most of the possible distributions of surface height errors, and obtain before and after wavefront control RMS wavefront errors from the statistical averages of those quantities.

\section{NUMERICAL RESULTS}

In this section, we present several numerical examples on the synthesis and the analysis of PSD errors using $M \Delta x=N \Delta y=1 \mathrm{~m}, p=3, \rho_{H P}=1 \mathrm{cyc} / \mathrm{m}, \sigma_{0}=100 \mathrm{~nm}, M=N=512$, and $Q=32$, where "cyc" means "cycle". Most people prefer to use (length) $)^{-1}$ for the unit of $\rho$, and (length $)^{2}(\text { length })^{2}$ for the unit of PSD. The former is the same 
as "cyc/length". We sometimes encounter a unit of "cyc/aper", meaning "cycle per aperture". As we know, aperture is always two-dimensional. But in the case of "cyc/aper" it is used as a measure of the width (or height) of a square aperture. This unit is sometimes more appealing, because " $n$ cyc/aper" means $n$ full-cycles of sinusoidal ripples across the clear aperture of an optical surface. With our choice of parameters listed above, we have $1 \mathrm{cyc} / \mathrm{m}=1 \mathrm{cyc} / \mathrm{aper}$, $u_{\max }=v_{\max }=256 \mathrm{cyc} /$ aper, $\rho_{H P}=1 \mathrm{cyc} /$ aper, and $\rho_{\max }=\sqrt{2} u_{\max }=362.04$ cyc/aper, where the subscript "max" means "maximum". Using a similar notation, the unit of PSD can also be expressed as (length) ${ }^{2}$ (aper) ${ }^{2}$ or simply (length) $)^{2} A$ or $\lambda^{2} A$, where $A$ is the area of a square surface as defined in Eq. (5) and $\lambda$ is the wavelength. For example, in the last case, $\mathrm{PSD}=10 \lambda^{2} A$ means $\mathrm{PSD}=(10$ wave-square $) \times($ surface area $)$.

\subsection{General Cases}

We now present some numerical examples. First, we examine the effect of a circular mask or a circular aperture on the PSD. Figure 1(a) shows one realization of $h^{\prime}\left(x_{m}, y_{n}\right)$ obtained from Eq. (14), and Fig. 1(b) shows the corresponding PSD data. As mentioned earlier, $h^{\prime}\left(x_{m}, y_{n}\right)$ corresponds to a square clear aperture. In Fig. 1(b), the 1D- $P S D_{\text {true }}$ curve is calculated from the $P S D^{\prime}\left(u_{m}, v_{n}\right)$ defined in Eq. (11), 2D- $P S D_{\text {calc }}$ are the 2D-PSD data obtained from $h^{\prime}\left(x_{m}, y_{n}\right)$ using Eq. (4), and $1 \mathrm{D}-P S D_{\text {calc }}$ is the radial average of the $2 \mathrm{D}-P S D_{\text {calc }}$. As we can see, both the $2 \mathrm{D}-P S D_{\text {calc }}$ and the $1 \mathrm{D}-$ $P S D_{\text {calc }}$ data fall on top of the $1 \mathrm{D}-P S D_{\text {true }}$ curve in this case as expected. Additional information is given about these figures in the figure caption.
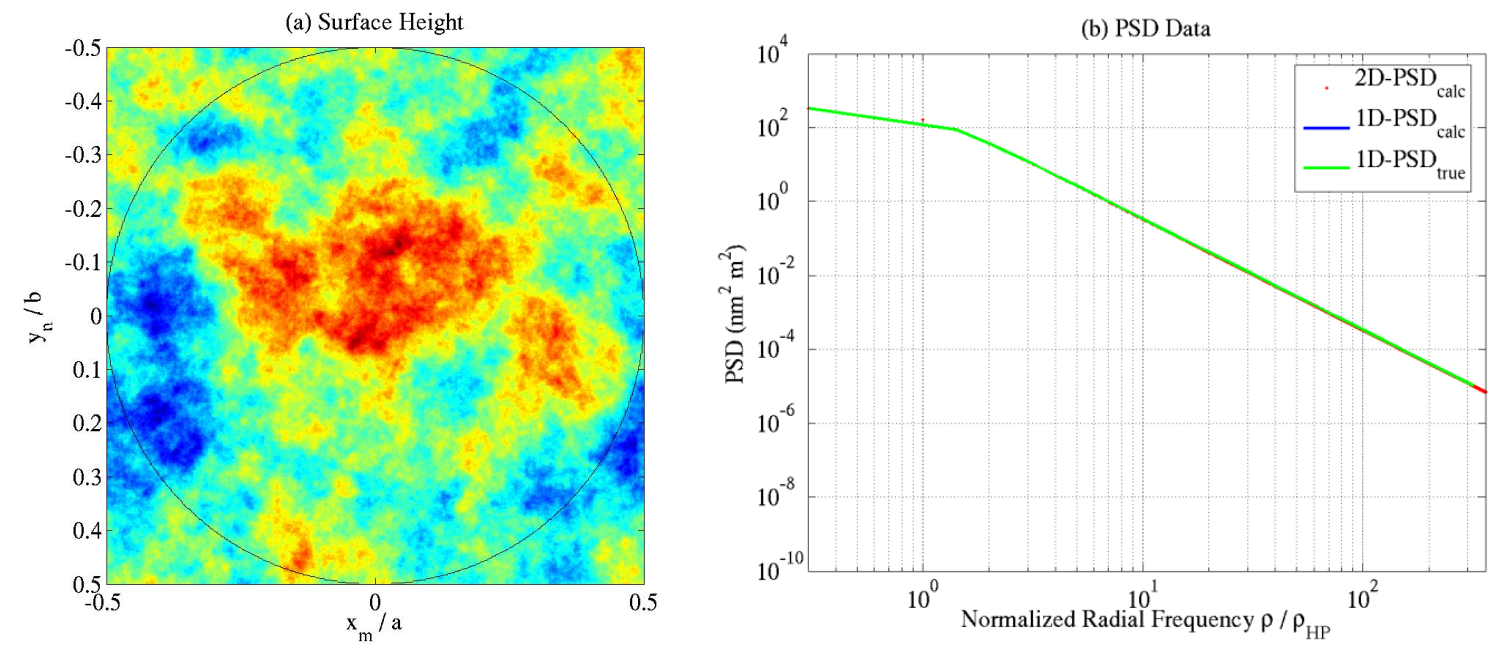

Figure 1. (a) One realization of $h^{\prime}\left(x_{m}, y_{n}\right)$ from Eq. (14), where the black-circle indicates the boundary of circular mask mask $\left(x_{m}, y_{n}\right)$ used in this study. (b) Corresponding PSD data. The value of $\rho_{00} / \rho_{\mathrm{HP}}$ is changed from 0 to 0.3 in part (b) to shorten the displayed length of the horizontal axis. In this case, $\sigma=100 \mathrm{~nm}$ and $\sigma_{\mathrm{mr}}=93.1 \mathrm{~nm}$, where the subscript "mr" means "mean-removed". The $\sigma_{\mathrm{mr}}$ is obtained from $h^{\prime}\left(x_{m}, y_{n}\right)$ after removing its mean value.

Figure 2(a) shows the PSD data obtained from $h\left(x_{m}, y_{n}\right)$ having a non-zero circular area defined by the black-circle in Fig. 1(a). The resultant 2D- $P S D_{\text {calc }}$ now consists of discrete data points scattered in the vicinity of the $1 \mathrm{D}-P S D_{\text {true }}$ curve. When we obtain $h^{\prime}\left(x_{m}, y_{n}\right)$ from $P S D_{\text {true }}$ first, then $P S D_{\text {calc }}$ from $h\left(x_{m}, y_{n}\right)=h^{\prime}\left(x_{m}, y_{n}\right) \times$ mask $\left(x_{m}, y_{n}\right)$, the $P S D_{\text {calc }}$ gives an RMS value different from that of $P S D_{\text {true }}$ or $\sigma_{0}$. In order to compare 2D- $P S D_{\text {calc }}$ with $2 \mathrm{D}-P S D_{\text {true }}$, we multiply $P S D_{\text {calc }}$ by a RMS scaling factor, $\gamma_{\text {rms }}$. The $\gamma_{\text {rms }}$ is defined as 

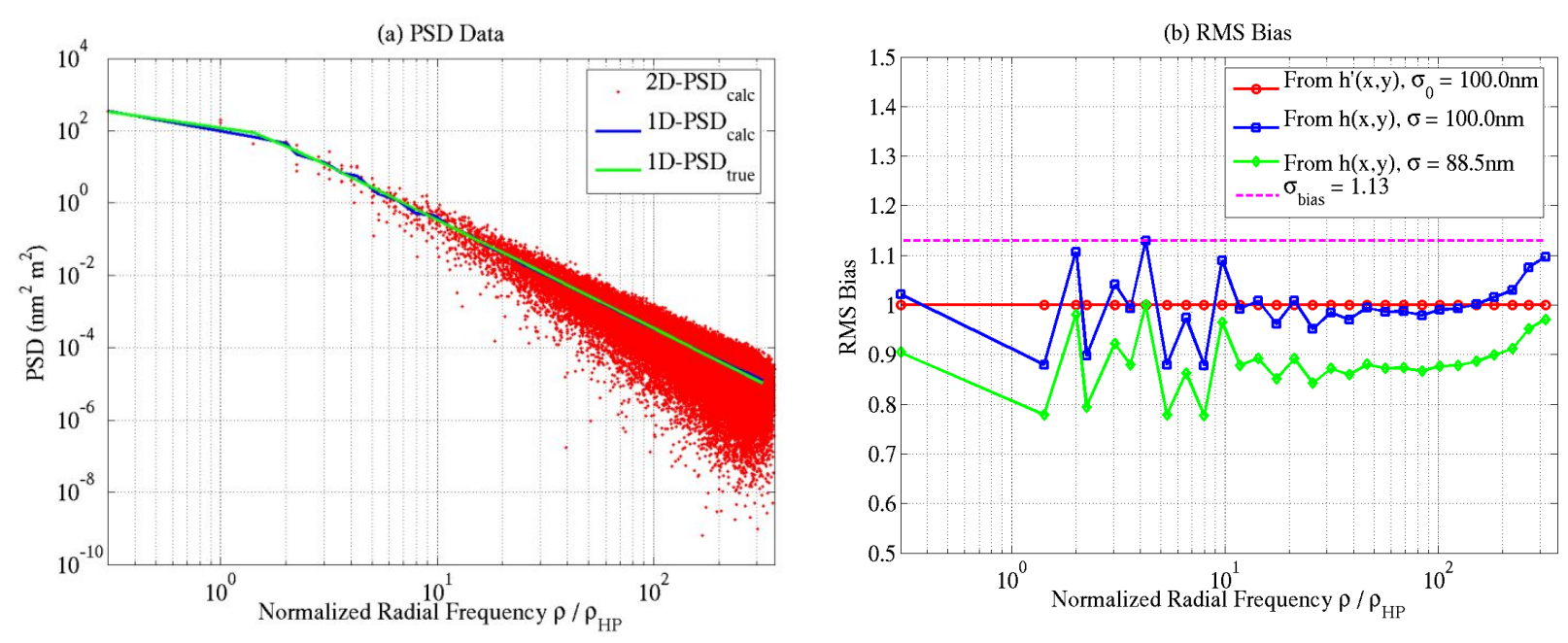

Figure 2. (a) Same as Fig. 1(b) except that these data were obtained from the masked surface height map $h\left(x_{m}, y_{n}\right)$ defined in Eq. (13). The original surface height map $h^{\prime}\left(x_{m}, y_{n}\right)$ is the same as in Fig. 1(a). In this case, $\sigma_{\text {aper }}=106.4 \mathrm{~nm}$ and $\sigma_{\mathrm{mr}}=96.5 \mathrm{~nm}$, respectively. The 2D-PSD calc data were rescaled before plotting so that they have $\sigma=\sigma_{0}$. (b) The

RMS bias $\sigma_{\text {bias }}$ corresponding to $h^{\prime}\left(x_{m}, y_{n}\right)$ and $h\left(x_{m}, y_{n}\right)$, respectively. The mean and the standard deviation (STD) of the blue-curve are 0.997 and 0.064 , respectively.

(a) RMS Values of $h(x, y)$

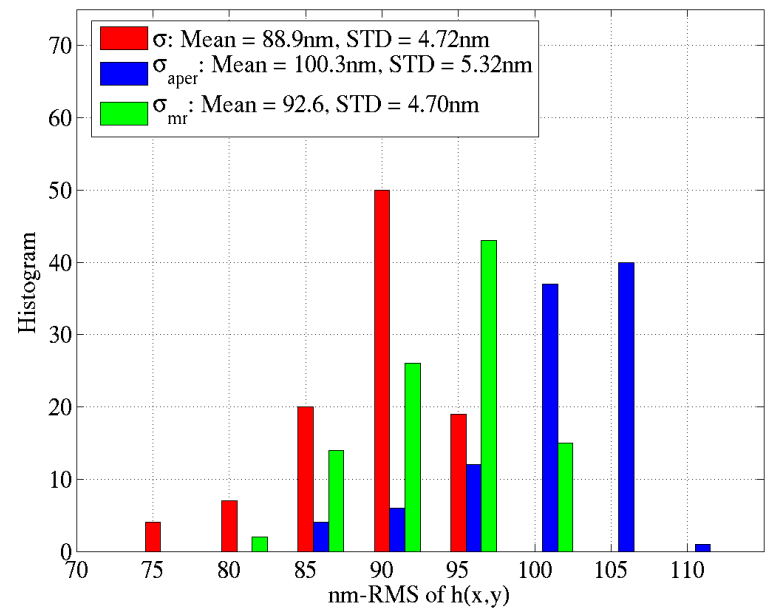

(b) Mean $=37.5 \mathrm{~nm}$, STD $=10.09 \mathrm{~nm}$

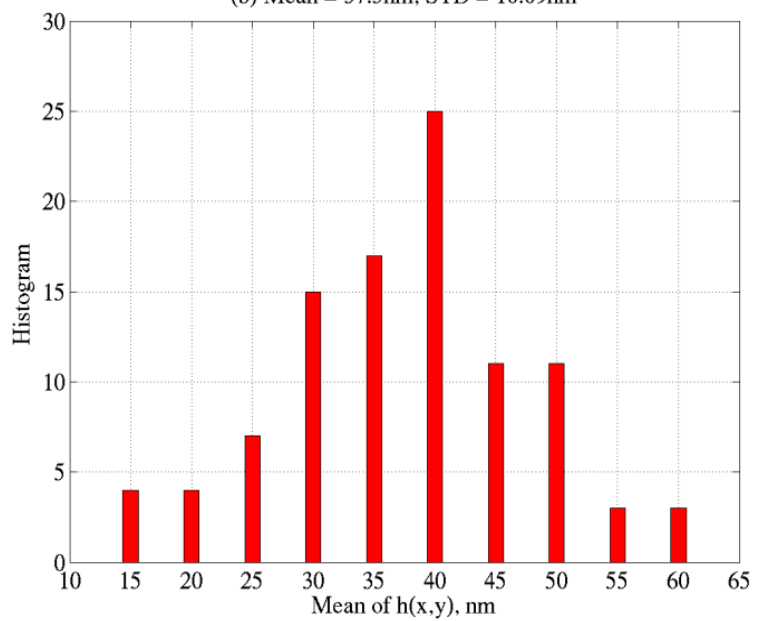

Figure 3. (a) Distributions of $\sigma, \sigma_{\text {aper }}$ and $\sigma_{\mathrm{mr}}$ values for $100 h\left(x_{m}, y_{n}\right)$ realizations. (b) Mean values of $h\left(r_{m n}\right)$ defined in Eq. (9). "STD” means "standard deviation".

$$
\gamma_{\mathrm{rms}}=\frac{\sigma_{0}}{\sigma}
$$

where $\sigma$ is defined in Eq. (8). As we can see from Fig. 2(a), the 1D- $P S D_{\text {calc }}$ shows a characteristic very similar to that of the 1D- $P S D_{\text {true }}$ curve. This is one of the most important findings of this study. That is, the scattering of the PSD data is caused in this simulated case only by the truncation of the surface height map to a circular non-zero area, but the radial average of these scattered PSD data still preserves the main characteristic of the true PSD function. We have 
$\sigma_{\text {aper }}=106.4 \mathrm{~nm}$ and $\sigma_{\mathrm{mr}}=96.5 \mathrm{~nm}$ in this case, where $\sigma_{\mathrm{mr}}$ is the mean-removed version of $\sigma_{\text {aper }}$. Figure 2(b) shows the RMS bias ${ }^{1}$ defined as

$$
\sigma_{\text {bias }}=\operatorname{RMS~Bias~}=\sqrt{P S D_{\text {calc }} / P S D_{\text {true }}} .
$$

In Fig. 2(b), the red and the blue curves were obtained from $h^{\prime}\left(x_{m}, y_{n}\right)$ and $\gamma_{\mathrm{rms}} h\left(x_{m}, y_{n}\right)$, respectively. What is shown here are the $\sigma_{\text {bias }}$ values of the 1D-PSD. The same calculation can be carried out on the 2D-PSD as well. It should be noted that the $1 \mathrm{D}-\sigma_{\text {bias }}$ can be less than 1 everywhere when $\sigma<\sigma_{0}$, that is, when the measured surface height RMS value is less than the specification. For example, in the case of Fig. 2(a), re-scaling $\sigma$ to $\sigma=88.5 \mathrm{~nm}$ makes $\sigma_{\text {bias }} \leq 1$ everywhere.

As we can see from the results in Figs. 1 and 2, the PSD specification of an optical surface can be given in terms of the following 3 parameters: (i) The RMS value of the $P S D_{\text {calc }}, \sigma$, relative to $\sigma_{0}$; (ii) the exponent of the inverse power law, $p$, determined from the 1D-PSD curve; and (iii) the RMS bias, $\sigma_{\text {bias }}$. For example, one can specify that the measured surface height map must have $\sigma \leq \sigma_{0}, p_{\text {meas }} \geq p_{\text {spec }}$, and $\sigma_{\text {bias }} \leq 1$, where the subscripts "meas" and "spec" mean "measured" and "specified", respectively. As is seen in the above example, one always gets $\sigma^{\prime}=\sigma_{0}$, but $\sigma_{\text {aper }}$ can be larger than, equal to or smaller than $\sigma_{0}$ when deriving the PSD specifications based on surface height maps obtained from Eqs. (14) and (13). In order to get a better understanding on the relationships among various RMS values, we calculated the $\sigma, \sigma_{\text {aper }}$ and $\sigma_{\mathrm{mr}}$ values of 100 PSD realizations obtained with $\sigma_{0}=100 \mathrm{~nm}$. The results are shown as histograms in Fig. 3(a). Figure 3(b) shows the histogram of the mean values of the corresponding $h\left(r_{m n}\right)$ explained in relation to Eq. (9) above.

Now let us look at another example, as shown in Figs. 4. In this case we used $p=2$ to obtain $P S D_{\text {true }}$, and obtained the $P S D_{\text {calc }}$ data from a different realization of the PSD error using the same parameters as in Fig. 1. We now have $\sigma_{\text {aper }}=$ $96.0 \mathrm{~nm}$, and $\sigma_{\text {bias }}<1$ in regions where $\rho_{\mathrm{mn}}>3 \rho_{\mathrm{HP}}$.
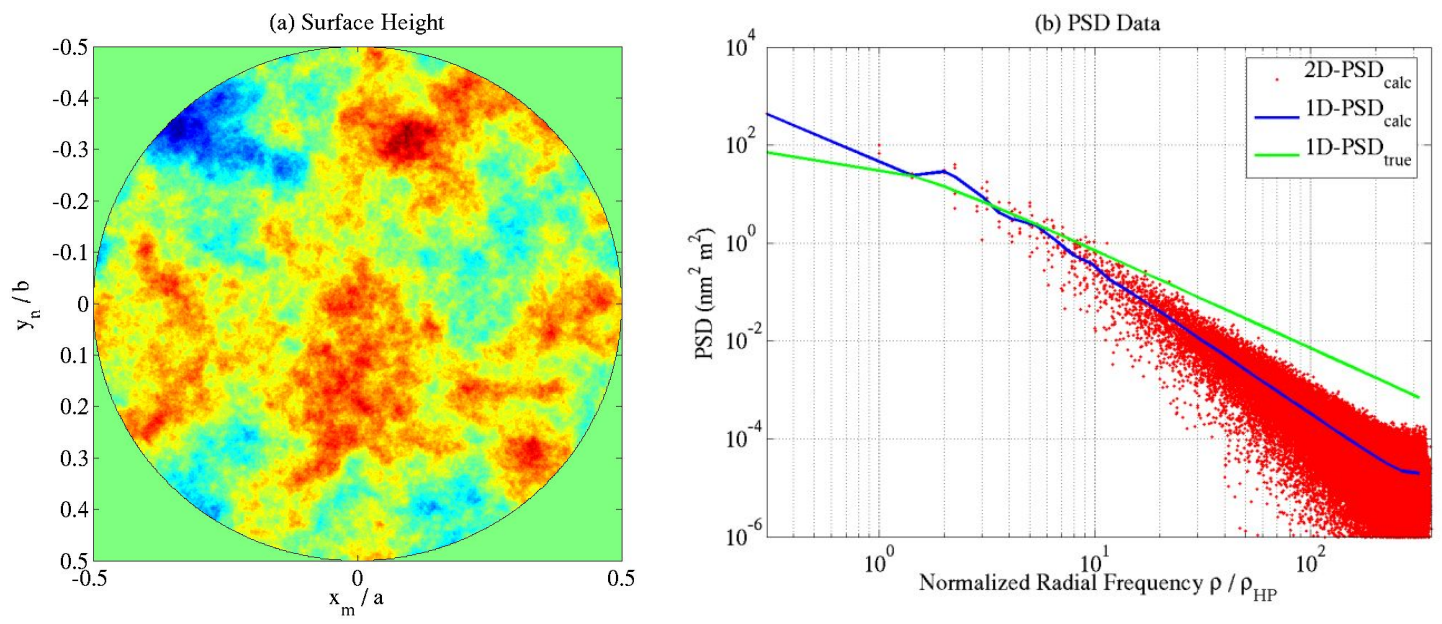

Figure 4. Same as Fig. 1 except that (i) $P S D_{\text {true }}$ was obtained with $p=2$; (ii) the $P S D_{\text {calc }}$ was obtained from a different realization of $h^{\prime}\left(x_{m}, y_{n}\right)$ with $p=3$. In this case, $P S D_{\text {calc }}$ has $\sigma_{\text {aper }}=96.0 \mathrm{~nm}$ and $\sigma_{\text {bias }}<1$ when $\rho_{m n}>3 \rho_{\mathrm{HP}}$. 


\subsection{Role of a Windowing Filter}

One of the preferred approaches used for reducing variance in the PSD data is to multiply a measured surface height map with a windowing filter, such as Hann or Welch window, in the spatial domain before calculating the 2D-PSD. In this sub-section, we examine the effects of a Welch window. A Welch window is defined as ${ }^{1}$
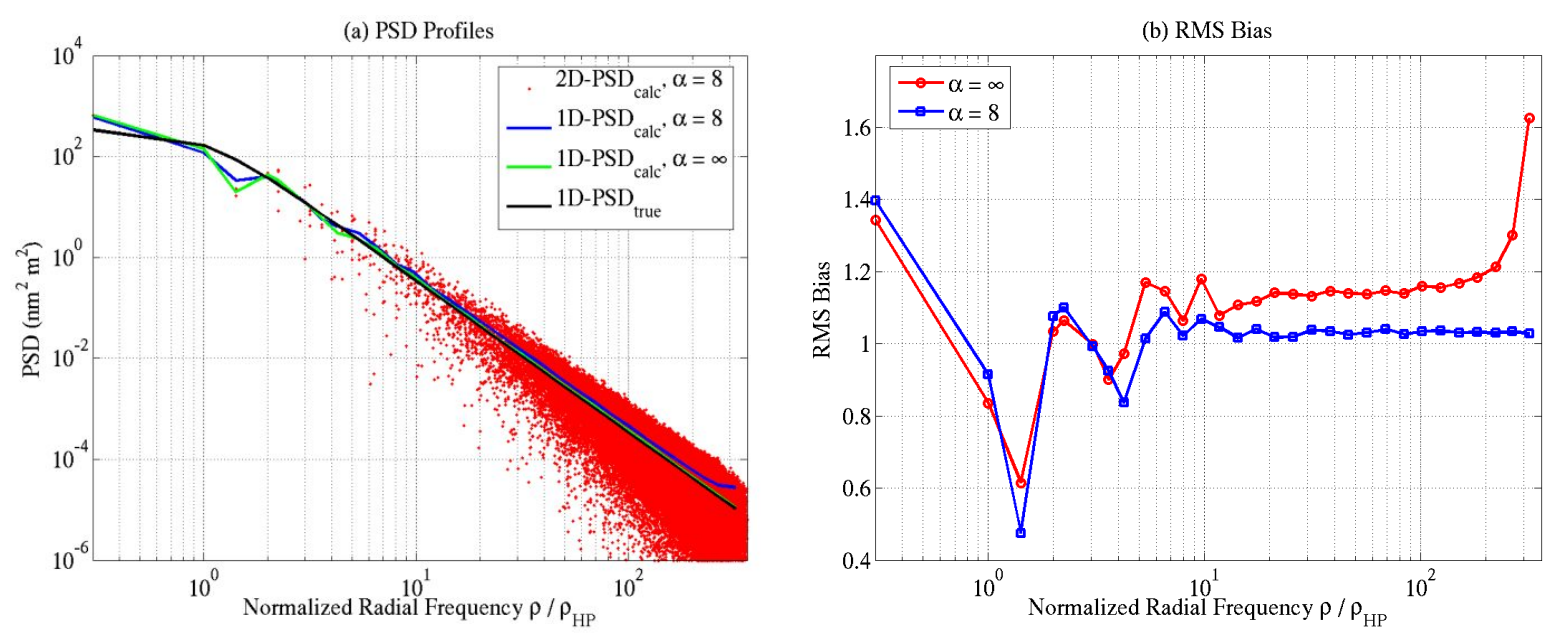

Figure 5. (a) PSD data corresponding to the PSD realization shown in Fig. 4(a). The both sets of 2D PSD calc data were rescaled so that their $\sigma$ values are the same as $\sigma_{0}$. (b) The corresponding $\sigma_{\text {bias }}$ curves obtained from the 1D-PSD data. $[$ Mean, STD $]=[1.119,0.166]$ for the red-curve and . $[$ Mean, STD $]=[1.016,0.133]$ for the blue-curve.

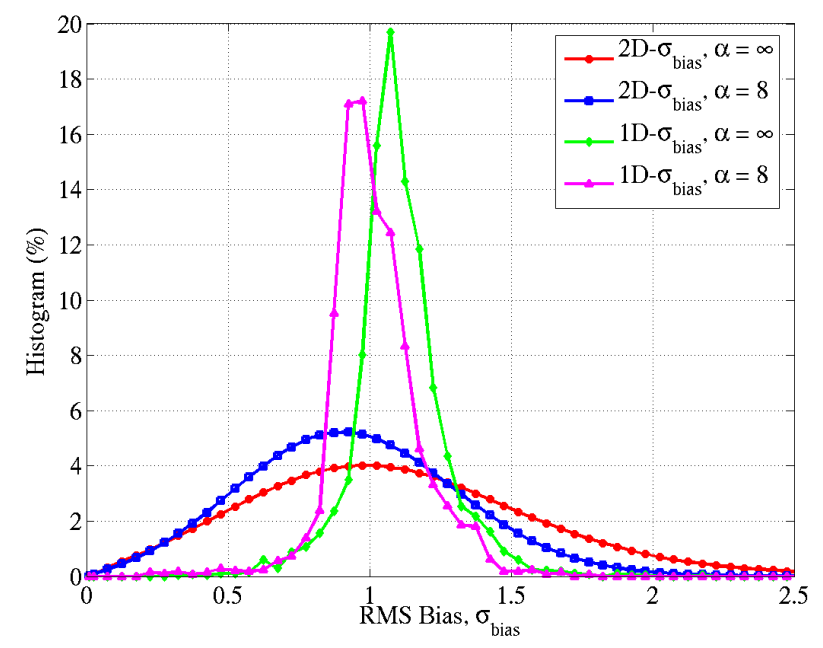

Figure 6. Histograms of $\sigma_{\text {bias }}$ obtained from 100 different PSD realizations for each of the $\alpha=\infty$ and the $\alpha=8$ cases, respectively. The peak of the red-curve is at $\sigma_{\text {bias }}=0.975$, and that of the blue-curve is at $\sigma_{\text {bias }}=0.925 . R M S\left(\sigma_{\text {bias }}\right.$ $-1)=0.190$ for the $1 \mathrm{D}-\sigma_{\text {bias }}$ with $\alpha=\infty$, and $\operatorname{RMS}\left(\sigma_{\text {bias }}-1\right)=0.160$ for the $1 \mathrm{D}-\sigma_{\text {bias }}$ with $\alpha=8$. 


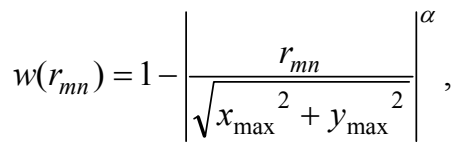

where $r_{m n}$ is defined in Eq. (9), and $x_{\max }$ and $y_{\max }$ are the maximum values of $x_{m}$ and $y_{n}$ inside the surface height map clear aperture. Detailed discussion of the above Welch window can be found in Ref. [1]. A Welch window index of $\alpha=8$ was found to represent a good compromise between smearing and leakage ${ }^{1}$ and we use that value in our simulations. In order to compare the 2D- $P S D_{\text {calc }}$ results of $h\left(x_{m}, y_{n}\right)$ and $h\left(x_{m}, y_{n}\right) \times w\left(r_{m n}\right)$ with 2D- $P S D_{\text {true }}$, we rescaled the former two 2D-PSDs so that they have the same RMS value as that of $2 \mathrm{D}-P S D_{\text {true }}$.

In Figs. 5(a) and 5(b), we compared the 1D- PSD profiles and the $\sigma_{\text {bias }}$ values of two cases, $\alpha=\infty$ and $\alpha=8$, respectively. The former case corresponds to the surface height map that is not multiplied by the windowing filter. The $h\left(x_{m}, y_{n}\right)$ used in this simulation is the same as in Fig. 4(a). Therefore, the green-curve in Fig. 5(a) is the same as the blue-curve in Fig. 4(b). As is evident from Fig. 5(b) as well as the mean and STD values of the two curves given in the figure caption, the application of the Welch window improves the $1 \mathrm{D}-\sigma_{\text {bias }}$ values in this example, but such improvement is not significant.

In order to gain better understanding about the role of the windowing filter, we repeated the above simulation for 100 $h\left(x_{m}, y_{n}\right)$ realizations for each of the $\alpha=\infty$ and the $\alpha=8$ cases, respectively, and obtained the histograms of the $\sigma_{\text {bias }}$ values shown in Fig. 6. In the cases of 2D-PSD, the histogram of the $\sigma_{\text {bias }}$ values is narrower for the case of $\alpha=8$ than for the $\alpha=\infty$ as expected, but its peak is at $\sigma_{\text {bias }}=0.925$, which is further away from the $\sigma_{\text {bias }}=1$ point relative to the peak of the $\alpha=\infty$ case, which is at $\sigma_{\text {bias }}=0.975$. In the cases of 1D-PSD, $R M S\left(\sigma_{\text {bias }}-1\right)=0.190$ for the $\alpha=\infty$ case, and $\operatorname{RMS}\left(\sigma_{\text {bias }}-1\right)=0.160$ for the $\alpha=8$. That is, applying a windowing filter to the $h\left(x_{m}, y_{n}\right)$ data improves the $\sigma_{\text {bias }}$ values of the 2D-PSD as expected, but for 1D-PSD such improvement is again not significant. This means that applying a Welch window to the $h\left(x_{m}, y_{n}\right)$ data in the current azimuthally-averaging method improves the PSD variance only marginally. This is another important finding of this study.

\subsection{Role of Welch Overlapping Subapertures}

It was found that Welch Overlapping Sub-Aperture (WOSA) 2D-PSD calculation on a synthesized mid-spatial frequency only surface reduces the 2D-PSD error, defined as $\triangle P S D=\left|P S D_{\text {true }}-P S D_{\text {calc }}\right| / P S D_{\text {calc }}$, by nearly an order of magnitude relative to that of the full-aperture PSD ${ }^{1}$. In this sub-section, we examine whether or not the WOSA 2DPSD calculation improves the $\sigma_{\text {bias }}$ calculated from the radially-averaged 1D-PSD.

In our example, we divide the $h\left(x_{m}, y_{n}\right)$ map into 8 overlapping subapertures as shown in Fig. 7(a) first, where the fullaperture $h\left(x_{m}, y_{n}\right)$ map is the same as in Fig. 4(a). This pattern provides 95\% areal coverage over the full circular aperture. Then we calculate the subaperture 1D-PSDs shown in Fig. 7(b) using the same MATLAB code used to calculate the full aperture, radially-averaged 1D-PSD. Again, we rescaled each 2D-PSD such that all of the 2D-PSDs have an RMS value same as that of the 2D- $P S D_{\text {true }}$. The WOSA 1D-PSD, shown as $P S D_{\text {avrg }}$ in Fig. 8(a), was calculated from the average of the subaperture 1D-PSDs shown in Fig. 7(b). Figure 8(b) shows the $\sigma_{\text {bias }}$ curves obtained from the three curves shown in Fig. 8(a) using Eq. (16) with $P S D_{\text {true }}$ as the denominator. As we can see from this figure as well as the mean and the STD values of two $\sigma_{\text {bias }}$ curves, the WOSA PSD calculation does not improve the $\sigma_{\text {bias }}$ values in the current, radially-averaged 1D-PSD approach. 


\subsection{Effects of Low-Order Figure Errors on Radially-Averaged 1D-PSD}

We now look at the effects of low-order figure errors on the radially-averaged 1D-PSD. Figure 9(a) shows a surface height map $z\left(x_{m}, y_{n}\right)$ calculated from Noll-order ${ }^{11}$ Zernike-polynomial coefficients $Z_{5}-Z_{10}$ whose values were listed in the figure caption. Nothing is special about the Zernike coefficients used here; they are just one random realization. Figure 9(b) shows the corresponding 1D-PSDs. The blue curve (Case 2) was obtained in the following way: We rescaled the $2 \mathrm{D}-P S D_{\text {calc }}$ so that in the absence of $z\left(x_{m}, y_{n}\right)$ it has the same RMS value as that of $2 \mathrm{D}-P S D_{\text {true }}$. Whereas the green curve (Case 3) corresponds to a case where the 2D- $P S D_{\text {calc }}$ of $z\left(x_{m}, y_{n}\right)+h\left(x_{m}, y_{n}\right)$, with a $h\left(x_{m}, y_{n}\right)$ map same as in Fig. 4(a), has the same RMS value as that of 2D- $P S D_{\text {true }}$. We can see from the blue-curve of Fig. 9(b) that the low-order figure errors leak into all of the frequency components in the PSD domain, and this is true even after the RMS value of the 2D- $P S D_{\text {calc }}$ of $z\left(x_{m}, y_{n}\right)+h\left(x_{m}, y_{n}\right)$ is adjusted so that it has the same RMS value as that of 2D$P S D_{\text {true }}$. This point is more evident in Fig. 9(c), where the red-curve (Case 1) was calculated from the 1D- $P S D_{\text {calc }}$ of $h\left(x_{m}, y_{n}\right)$ only, the blue-curve (Case 2) from that of $z\left(x_{m}, y_{n}\right)+h\left(x_{m}, y_{n}\right)$ after re-scaling the corresponding 2D-PSD with $\gamma_{\text {rms }}$ of Case 1, and the green-curve (Case 3) from that of $z\left(x_{m}, y_{n}\right)+h\left(x_{m}, y_{n}\right)$ after re-scaling the corresponding 2D-PSD with its own $\gamma_{\mathrm{rms}}$ factor. These results show that it is necessary to remove all of the low-order figure errors from $h\left(x_{m}, y_{n}\right)$ before carrying out the PSD calculation to estimate the mid- and the high-frequency PSD components accurately.
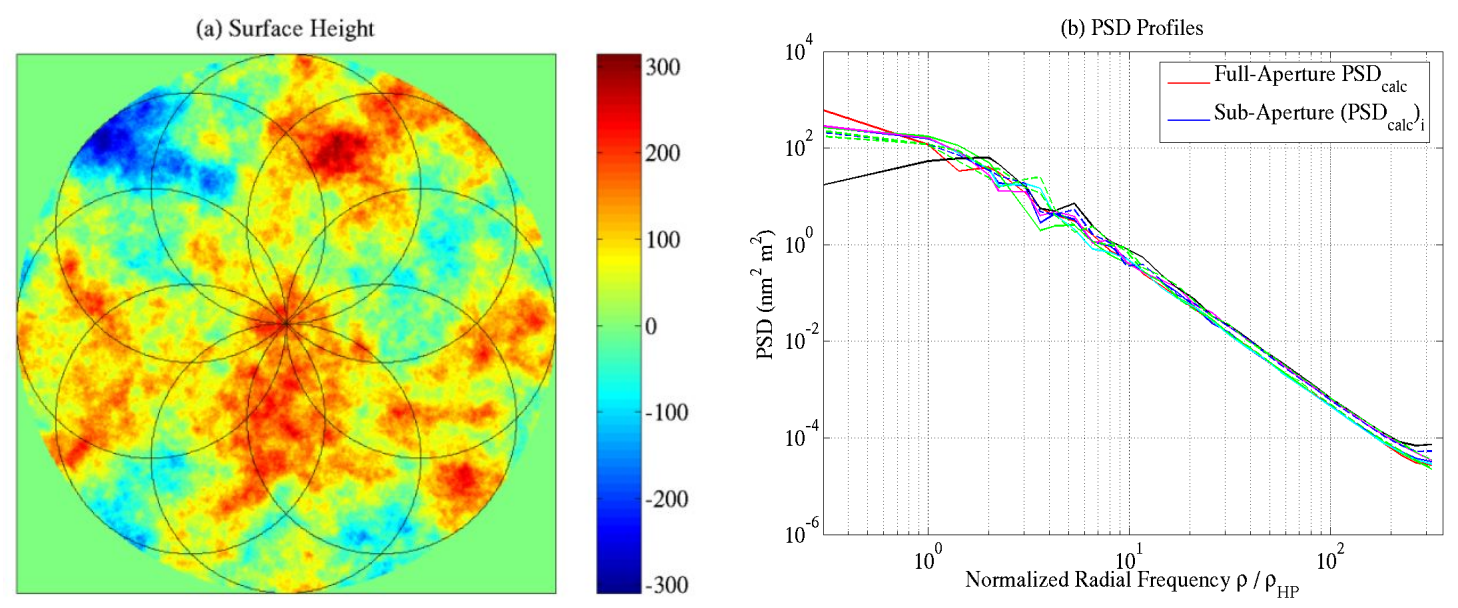

Figure 7. (a) The overlapping 8-subaperture pattern overlaid on a $h\left(x_{m}, y_{n}\right)$ map. This $h\left(x_{m}, y_{n}\right)$ map is the same as in

Fig. 4(a). (b) Radially averaged subaperture PSDs obtained from the 8 subaperture $\gamma_{\mathrm{rms}} h\left(x_{m}, y_{n}\right)$ maps shown in part (a). The full-aperture $1 \mathrm{D}-P S D_{\text {calc }}$ is also included for comparison. Only one subaperture $P S D_{\text {calc }}$ is included in the figure legend. 

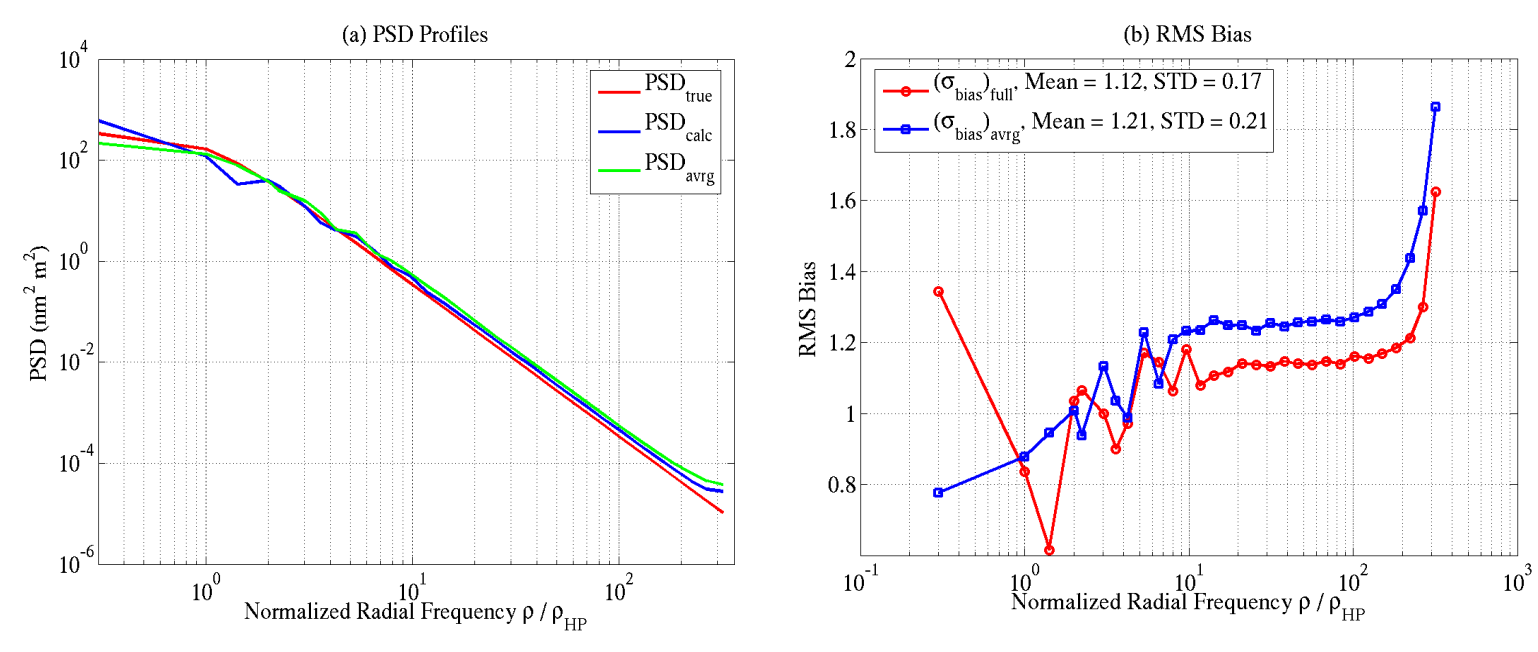

Figure 8. (a) Comparison of three radially averaged 1D-PSD curves, where $P S D_{\text {avrg }}$ is the average of 8 subaperture PSD curves shown in Fig. 7(b). (b) The corresponding 1D- $\sigma_{\text {bias }}$ curves, where $\left(\sigma_{\text {bias }}\right)_{\text {full }}$ is obtained from $P S D_{\text {calc }}$ and $P S D_{\text {true }}$, and $\left(\sigma_{\text {bias }}\right)_{\text {avrg }}$ from $P S D_{\text {avrg }}$ and $P S D_{\text {true }}$ shown in part (a).
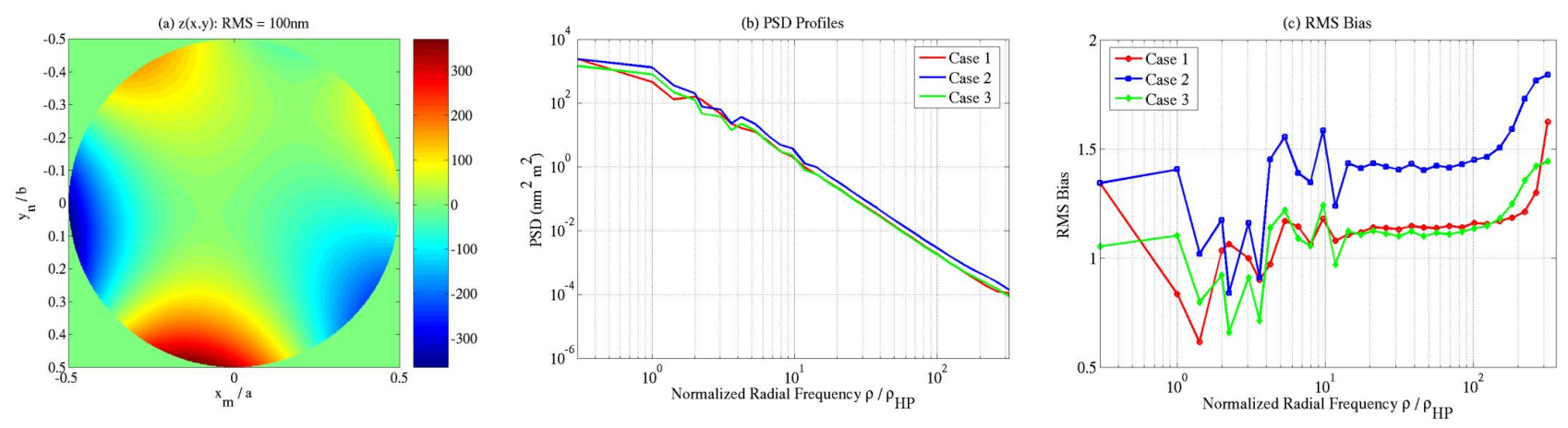

Figure 9. (a) Surface height map $z\left(x_{m}, y_{n}\right)$ obtained from the following Zernike-polynomial (Noll-order) coefficients: $z_{5}=-$ 18.0, $\mathrm{z}_{6}=-69.2, \mathrm{z}_{7}=5.2, \mathrm{z}_{8}=12.0, \mathrm{z}_{9}=-47.6$, and $\mathrm{z}_{10}=49.5 \mathrm{~nm}$. These Zernike-polynomial coefficients correspond to one random realization. (b) 1D-PSD profiles. The $h\left(x_{m}, y_{n}\right)$ used in this simulation is the same as in Fig. 4(a). Case 1 was obtained from $h\left(x_{m}, y_{n}\right)$ by rescaling the corresponding 2D-PSD calc with $\gamma_{\mathrm{rms}}$ such that it has the same RMS value as that of $2 \mathrm{D}-P S D_{\text {true }}$, that is, $\sigma=\sigma_{0}$. Case 2 was obtained from $h\left(x_{m}, y_{n}\right)+z\left(x_{m}, y_{n}\right)$ by multiplying the resultant 2D- $P S D_{\text {calc }}$ with $\gamma_{\text {rms1 }}$. Case 3 was obtained from $h\left(x_{m}, y_{n}\right)+z\left(x_{m}, y_{n}\right)$ by multiplying the resultant 2D$P S D_{\text {calc }}$ with its own $\gamma_{\text {rms }}$ such that its $\sigma=\sigma_{0}$. (c) The corresponding $\sigma_{\text {bias }}$ curves.

\section{CONCLUSION}

The calculation of PSD errors corresponding to a surface height map always requires a rectangular or square surface height data matrix. But in most real cases the surface height map is defined inside a circular aperture and its matrix elements outside that circular aperture are all filled with zeros. We have shown by numerically evaluating the PSD errors of several synthesized surface height maps that such zero-elements of the surface height map by themselves only cause the scattering of the PSD data when plotted as a function of radial frequency. To convert such scattered 2D-PSD data into a new set of smoothly-varying 1D-PSD that can be directly compared with a PSD function used to derive PSD specifications, we proposed a new method: Obtaining 1D-PSD from the radial average of the scattered 2D-PSD. We 
have shown that this approach also eliminates the need for existing variance-reducing methods, such as applying a windowing filter to the surface height map before the calculation of its PSD, as well as for dividing a surface height map into several overlapping subapertures, calculating the individual 2D-PSDs, and then averaging those individual PSDs to obtain the full-aperture PSD. We have also shown that it is necessary to remove all of the low-order figure errors from the surface height map before carrying out the PSD calculation to estimate the mid- and the high-frequency PSD components accurately. We have tried to provide a set of complete and easy-to-use formulations for the calculation of the PSD from a 2D surface height map, such as the one measured interferometrically, as well as for the synthesis of surface height maps from a PSD function needed in the process of deriving PSD requirements. We expect that this paper will serve as a valuable reference for the PSD analysis of optical surfaces, especially for beginners in this field.

\section{ACKNOWLEDGEMENTS}

The author is very grateful to Eri J. Cohen for providing him with several valuable references, to Jeffrey W. Yu and Serge Dubovitsky for their valuable inputs on the PSD formulations, and to Scott A. Basinger for proof-reading the manuscript. All of the people mentioned above are at Jet Propulsion Laboratory, California Institute of Technology.

\section{REFERENCES}

[1] J. E. Hayden and D. A. Content, "PSD data analysis and algorithm development," SPIE Optifab 2007 Tech. Digest, paper TD04-27 (2007).

[2] David A. Content, et. al, "Engineering trade studies for the Terrestrial Planet Finder-Coronagraph primary mirror," Proc. SPIE 5867, 58670X (2005).

[3] J. M. Bennett and L. Mattsson, Introduction to Surface Roughness and Scattering, Optical Society of America, Washington, D.C., 44-50 (1989).

[4] J. E. Harvey, "X-ray optics," in Handbook of Optics, $2^{\text {nd }}$ Edition, Optical Society of America, Washington, D.C., Volume 2, Chapter 11, 11.22-11.25 (1995).

[5] J. M. Elson and J. M. Bennett, "Calculation of the power spectral density from surface profile data," Appl. Optics 34, 201-208 (1995).

[6] C. J. Walsh, A. J. Leistner, and B. F. Oreb, "Power spectral density analysis of optical substrates for gravitationalwave interferometry," Appl. Optics 38, 4790-4801 (1999).

[7] R. N. Youngworth, B. B. Gallagher, and B. L. Stamper, "An overview of power spectral density (PSD) calculations," Proc. SPIE 5869, 58690U (2005).

[8] J. K. Lawson, C. R. Wolfe, K. R. Manes, J. B. Trenholme, D. M. Aikens, and R. E. English, Jr., "Specification of optical components using the power spectral density function," Proc. SPIE 2536, 38 (1995).

[9] Joseph J. Green, Jet Propulsion Laboratory, California Institute of Technology, Pasadena, California 91109 (personal communications, 2004).

[10] E. Sidick, S. A. Basinger, and D. C. Redding, "An improved wavefront control algorithm for large space telescopes," Proc. SPIE 7015, 70154P (2008).

[11] Robert J. Noll, “Zernike polynomials and atmospheric turbulence," J. Opt. Soc. Am. 66, pp. 207-211 (1976). 